
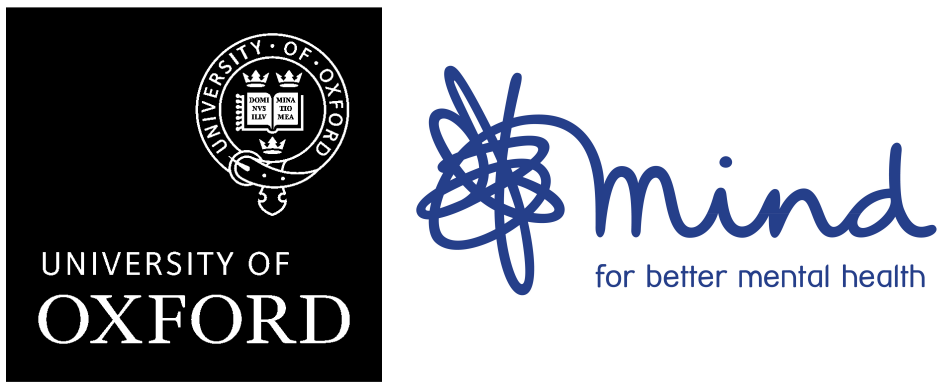

Lead researcher: Dr Jennifer Wild Department of Experimental Psychology Tinbergen Building

9 South Parks Road

Email: jennifer.wild@psy.ox.ac.uk

Please direct all enquiries to the research assistant: Gabriella Tyson Tel: 01865618610

Email: gabriella.tyson@psy.ox.ac.uk

Date: 01 October 2016

\title{
Participant Information Sheet \\ An evaluation of a new resilience intervention for emergency workers
}

We would like to invite you to take part in a study of a new resilience intervention developed for emergency workers. Before you decide, it is important for you to understand why the research is being done and what it will involve. Please take the time to read the following information carefully. You can email or telephone the researcher to ask any questions you may have about this study. If you are happy to participate, you will have the opportunity to ask further questions by email or telephone, and sign a consent form to join the study.

\section{Background and aims of the study}

We have developed a tailor-made course for emergency workers to help improve resilience and wellbeing. The course is free and is being delivered by your local Mind and the University of Oxford.

We are running it in two different ways because we want to measure which approach works best: accessing a mixed digital and weekly group format or an online only format, which can be accessed in the comfort of your own home.

\section{Why have I been asked to take part?}

You have been identified as a potential participant because you are employed by or volunteer for the police, fire and rescue, ambulance, or search and rescue emergency services.

\section{Do I have to take part?}

You do not have to take part in this research. Participation is voluntary and it is entirely up to you to decide whether or not you want to take part. Talk about it with friends and relatives if you wish, you may ask the researchers any questions about the study before making a decision about it. If you decide to take part, you will be asked to sign a consent form. You are free to leave the study at any time, without any penalty and without giving a reason.

What will happen in the study?

At the beginning of the study, after signing a consent form you will be asked to fill in questions about depression and anxiety. You will not be able to take part if these questionnaires suggest that you may have one of these problems, and would also benefit from treatment. If this is the case, the researcher will talk with you and give you suggestions 

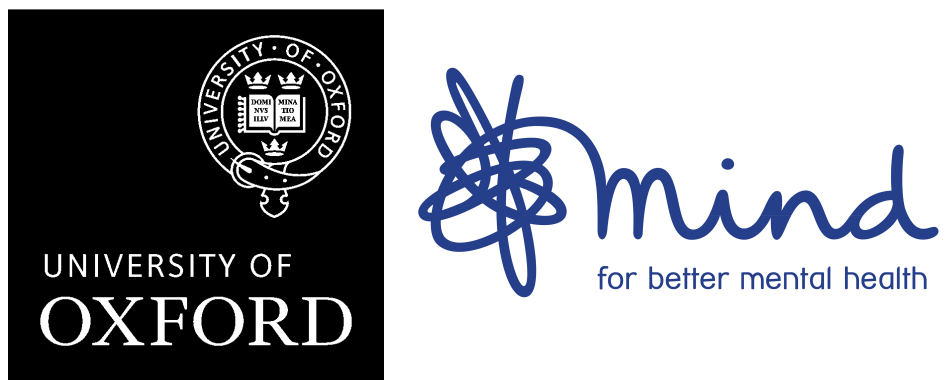

Lead researcher: Dr Jennifer Wild Department of Experimental Psychology

Tinbergen Building

9 South Parks Road

Email: jennifer.wild@psy.ox.ac.uk

Please direct all enquiries to the research assistant: Gabriella Tyson Tel: 01865618610

Email: gabriella.tyson@psy.ox.ac.uk

about what may be helpful. This could be a visit to your GP or accessing other local services or both. The screening questionnaires will be destroyed after use.

You will be able to take part if the questionnaires suggest that you do not have depression or post-traumatic stress. You will then be randomly allocated to one of three conditions: the mixed digital and group intervention, the digital-only intervention, or the delayed intervention, which means you would receive the mixed digital and group intervention after first waiting for four months. The interventions last four weeks. The decision about which intervention you will receive will be made by chance.

The mixed digital and group intervention requires up to two and a half hours per week. There is a module to complete online which takes about 20 minutes per week plus a group meeting, which is two hours in length and includes a break. It will take place in one of the four Mind sites: Peterborough and Fenland, Tyneside, Wirral, or London (City, Hackney and Waltham Forest). You will be invited to attend the site that is closest to you.

The digital only intervention requires about 30 minutes per week to complete online modules. One module is released per week for four weeks. You will be able to access this information in the comfort of your own home

The delayed intervention would require a waiting period of four months, with no active participation. You would then receive the mixed digital and group intervention, which would last four weeks as detailed above.

Over the course of the interventions, we will ask you to complete a number of questionnaires via a secure digital programme at three time points: before the intervention, after the intervention, and at three month follow-up. The questionnaires are short and will take about 30 minutes to complete before the intervention, and 20 minutes at postintervention and follow-up. We will also ask you to complete a very brief questionnaire every week. A sub-sample of 20 of our participants will be invited for in-depth interviews post-intervention.

The mixed digital and group intervention will be audio-recorded. We are only interested in recording the facilitator rather than your own contributions. We are audio-recording the facilitator to ensure that the intervention is being administered to the same standard at the different study sites. The audio memory files are confidential and will be securely transferred to our desktop at the University of Oxford. All audio files will be deleted once the study is completed.

If you participate in an in-depth interview after the intervention is over, your interview will be audio-recorded so that the researcher can transcribe the interview once it is over. The 

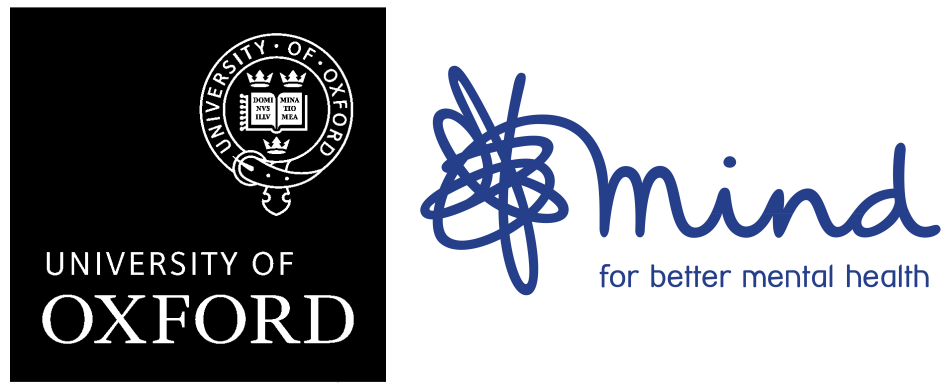

Lead researcher: Dr Jennifer Wild Department of Experimental Psychology

Tinbergen Building

9 South Parks Road

Email: jennifer.wild@psy.ox.ac.uk

Please direct all enquiries to the research assistant: Gabriella Tyson Tel: 01865618610

Email: gabriella.tyson@psy.ox.ac.uk

audiotapes are confidential and will be kept in locked cabinets at the University and destroyed once the study is completed.

\section{Are there any potential risks in taking part?}

There are no risks associated with taking part. However, there are significant benefits. Your participation in this trial could lead to your resilience being improved and your participation will help us in evaluating the resilience intervention, which will guide improvements to the course before it is made nationally available.

\section{What happens to the research data provided?}

All information you provide will be strictly confidential. Your name will be removed from your questionnaires and interview responses, and the anonymised research data will be stored on computers at the University of Oxford. Only the researchers and employees of Mind associated with the study will have access to the anonymised data. The data will be given a unique code that will be used in all subsequent data analysis and does not contain your name or any other personal information. Therefore, when the data are analysed the researchers will be unaware of who the data came from. Once the study is completed, the anonymised data will be stored on the UK data archive.

\section{Will the research be published?}

The results from this study may be published within the next 6 years. You will not be personally identified in any literature. However if you give your consent, anonymised quotes may be included in any report of publication that may arise from this research. You can obtain a copy of any publications from the contact numbers below.

\section{Who has reviewed this project?}

This project has been reviewed by, and received ethics clearance through, the University of Oxford Central University Research Ethics Committee.

\section{Who do I contact if I have a concern about the study or I wish to complain?}

If you have a concern about any aspect of this project, please speak to the research assistant Gabriella Tyson (01865 618610), the lead researcher Dr Jennifer Wild (01865 618600) or Jacob Diggle, Mind's senior research and evaluation officer (020 8215-2379) who will do their best to answer your query. The researcher should acknowledge your concern within 10 working days and give you an indication of how he/she intends to deal with it. If you remain unhappy or wish to make a formal complaint, please contact the chair of the Research Ethics Committee at the University of Oxford (using the contact details below) who will seek to resolve the matter in a reasonably expeditious manner:

Chair, Medical Sciences Inter-Divisional Research Ethics Committee; Email: ethics@medsci.ox.ac.uk; Address: Research Services, University of Oxford, Wellington Square, Oxford OX1 2JD 